\title{
Characterization of 2,3-diarylxanthones by electrospray mass spectrometry: gas-phase chemistry versus known antioxidant activity properties
}

\author{
Eduarda M. P. Silva ${ }^{1 *, \dagger}$, Cristina M. R. F. Barros ${ }^{2}$, Clementina M. M. Santos ${ }^{1,3}$, \\ António S. Barros ${ }^{1}$, M. Rosário M. Domingues ${ }^{2}$ and Artur M. S. Silva ${ }^{1 * *}$ \\ ${ }^{1}$ Organic Chemistry Group, QOPNA, Department of Chemistry, University of Aveiro, 3810-193 Aveiro, Portugal \\ ${ }^{2}$ Mass Spectrometry Centre, QOPNA, Department of Chemistry, University of Aveiro, 3810-193 Aveiro, Portugal \\ ${ }^{3}$ Department of Vegetal Production and Technology, Polytechnic Institute of Bragança, School of Agriculture, 5301-855 Bragança, \\ Portugal
}

RATIONALE: Xanthones $(\mathrm{XH})$ are a class of heterocyclic compounds widely distributed in nature that hold numerous noteworthy biological and antioxidant activities. Therefore, it is of utmost importance to achieve relevant detailed structural information to understand and assist prediction of their biological properties. The potential relationship between radical-mediated xanthone chemistry in the gas phase and their promising antioxidant activities has not been previously explored.

METHODS: Protonated xanthones XH1-9 were generated in the gas phase by electrospray ionization (ESI) and the main fragmentation pathways of the protonated XH1-9 formed due to collision-induced dissociation (CID) were investigated. RESULTS: In the CID-MS/MS spectra of $[\mathrm{M}+\mathrm{H}]^{+}$ions of $\mathrm{XH1}, \mathrm{XH} 2$ and $\mathrm{XH} 4$ the product ions formed due to $\mathrm{H}_{2} \mathrm{O}$ elimination corresponding to the base peak of the spectra. For the remaining six xanthones (XH3, XH5-9), showing the most promising biological profile, the product ion produced with the highest relative abundance (RA) corresponded to the one formed through concomitant loss of $\mathrm{H}_{2} \mathrm{O}$ plus $\mathrm{CO}$. Indicative of an inexistent or lower biological activity is the combined loss of $\mathrm{CO}$ plus $\mathrm{O}$ unique to the CID-MS/MS spectra of $\mathrm{XH1}, \mathrm{XH} 2, \mathrm{XH} 4$, and $\mathrm{XH}$. The product ion formed by loss of $64 \mathrm{Da}$ (concomitant loss of two molecules of $\mathrm{H}_{2} \mathrm{O}$ plus $\mathrm{CO}$ ) is only observed for xanthones containing a catechol unit (XH3 and XH6-9). This product ion has the highest RA for the most potent scavenger of reactive oxygen and nitrogen species XH9 that contains two of these catechol moieties.

CONCLUSIONS: A strong relationship between some of the biological activities of the studied 2,3-diarylxanthones and their ESI-MS/MS fragmentation spectra was found. The multivariate statistical analysis results suggest that the selected MS features are related to the important biological features. Copyright @ 2016 John Wiley \& Sons, Ltd.

Xanthones are a class of oxygen-containing heterocyclic compounds widely distributed in nature. ${ }^{[1]}$ They occur in two major plant families, Guttiferae and Gentianaceae, and in some families of fungi and lichens. ${ }^{[2]}$ The natural derivatives can present different substituents in the xanthone core namely hydroxyl, methoxyl, prenyl and glycosyl groups. These compounds hold a variety of noteworthy biological activities,

* Correspondence to: E. M. P. Silva, UCIBIO, REQUIMTE, Department of Chemistry, Faculty of Pharmacy, University of Porto, Rua de Jorge Viterbo Ferreira, 228, 4050-313 Porto, Portugal.

E-mail: esilva@ff.up.pt

** Correspondence to: A. M. S. Silva, Organic Chemistry Group, QOPNA, Department of Chemistry, University of Aveiro, 3810-193 Aveiro, Portugal.

E-mail: artur.silva@ua.pt

+ Present address: UCIBIO, REQUIMTE, Department of Chemistry, Faculty of Pharmacy, University of Porto, Rua de Jorge Viterbo Ferreira, 2284050-313 Porto, Portugal. including anti-allergic, anti-inflammatory and antitumor activities. ${ }^{[3]}$ In addition, a strong antioxidant activity has been demonstrated for these natural products, including the scavenging of reactive oxygen species (ROS) and reactive nitrogen species (RNS), as well as metal chelating effects. ${ }^{[4]}$ Their strong antioxidant activity is attributed to the presence of phenol and/or a catechol moieties at key positions.

Studies on the ability of synthetic hydroxylated 2,3diarylxanthones (Fig. 1) for scavenging ROS and $\mathrm{RNS}\left(\mathrm{O}_{2}^{\circ-}\right.$, $\mathrm{H}_{2} \mathrm{O}_{2},{ }^{1} \mathrm{O}_{2}, \mathrm{ROO}^{\circ}, \mathrm{HOCl}, \mathrm{NO}^{\bullet}$, and $\mathrm{ONOO}^{-}$) have been performed using chemical methods. The obtained results revealed that the tested 2,3-diarylxanthones $(\mathrm{XH})$ are outstanding scavengers of these species and nanomolar to micromolar ranges of $\mathrm{IC}_{50}$ values were found. The scavenging activity assayed using a non-enzymatic generating system revealed that the results seem to be largely affected by the number and position of the hydroxyl groups in the D and $\mathrm{E}$ rings of the xanthone core. ${ }^{[5,6]}$

Xanthone XH9, with two catechol moieties, was the most potent scavengers of all tested ROS and RNS. Also, from these studies it was possible to conclude that the $\mathrm{ROO}^{\bullet}$ scavenging 


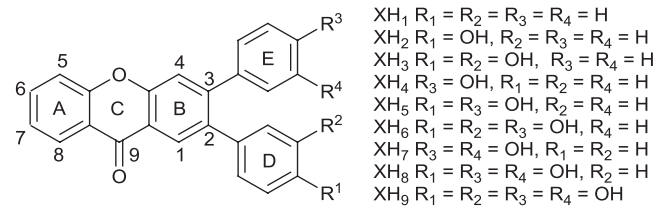

Figure 1. Structures of the studied xanthones XH1-9.

activity seems to depend mostly on the presence of phenolic groups while for the other tested ROS and RNS, xanthones with a catechol moiety were the most potent scavengers. ${ }^{[6]}$ For example, the most effective scavenger of $\mathrm{O}_{2}^{--}$was compound XH9 (Fig. 1), bearing four hydroxyl groups, followed by XH6 and XH8, which possess three hydroxyl groups. These studies showed that the presence of a catechol group in the D-ring is more relevant than a catechol group in the E-ring since the scavenging activity of XH6 is slightly higher than $\mathrm{XH}^{\left[{ }^{[6]}\right.}$ The HOCl-induced oxidation of dihydrorhodamine 123 (DHR) to rhodamine 123 was also efficiently prevented by all the tested 2,3-diarylxanthones. Interestingly, $\mathrm{XH} 5$, possessing two hydroxyl groups but not a catechol moiety, presents almost $100 \%$ inhibition of $\mathrm{HOCl}-$ induced oxidation at a concentration of $100 \mu \mathrm{M}^{[6]}$ At lower concentrations the scavenging effect of these xanthones seems to be more dependent on the presence of a catechol group rather than the number of hydroxyl groups.

The antioxidant and redox properties of these rather hydrophobic molecules have also been studied in a model multiphase aqueous medium, which mimics the complexity of the conditions inherent to biological structures. ${ }^{[7]}$ It has been observed in these model biological systems that a combination of hydrophobicity and steric factors also governs the antioxidant effectiveness of these molecules. ${ }^{[7]}$ For example, while XH9 is the least effective member of this series in the inhibition of $\mathrm{Cu}^{2+}$-induced lipid peroxidation in human low density lipoproteins (LDL), it is the most efficient scavenger of $\mathrm{O}_{2}^{\circ-}$ and halogenated peroxyl radicals in aqueous micellar systems. Due to its four hydroxyl groups, XH9 may be located preferentially in LDL towards the surface of the water-rich outer layer comprised of phospholipids, cholesterol and ApoB-100 apolipoprotein. By contrast, the more hydrophobic xanthones bearing two $\mathrm{OH}(\mathrm{XH} 3$ and $\mathrm{XH} 7)$ or three $\mathrm{OH}$ (XH6 and $\mathrm{XH} 8$ ) are most effective against LDL lipid peroxidation and they are expected to distribute more deeply into the phospholipid layer where the labile hydrogen sites participating in peroxidative chain reactions are found. Xanthones bearing an $\mathrm{OH}(\mathrm{XH} 2$ and $\mathrm{XH} 4)$ at the para position of their D or E phenyl rings are unreactive. ${ }^{[7]}$

The flavonoid quercetin is known to reduce the $\alpha$ tocopheroxyl radical ( $\left.{ }^{\bullet} \mathrm{TocO}\right)$ and reconstitute $\alpha$-tocopherol (TocOH). The dependence of $\alpha$-tocopheroxyl radical reduction by structurally related hydroxy-2,3-diarylxanthones (Fig. 1) on structure and micro-environment has also been evaluated using pulse radiolysis. ${ }^{[8]}$ These polyphenolic compounds exhibited antioxidant activity which exceeds that of quercetin in biological systems. It has been shown that, among the studied xanthones, only $\mathrm{XH} 9$ reduces ${ }^{\bullet} \mathrm{TocO}$, although repair depends strongly on the micro-environment. ${ }^{[8]}$

Electrochemical methodologies were also applied to establish correlations between structure and oxidation potential of these phenol- and catechol-substituted 2,3diarylxanthones XH1-9. Cyclic voltammetry was the electroanalytical technique used to clarify the electrochemical behaviour of these molecules. The results obtained were compared with the scavenging activities for ROS and RNS and once more the number and position of the hydroxyl substituents were determinant for the electrochemical behaviour of these molecules. Oxidation mechanisms were proposed and the electrochemical profiles of these xanthones corroborated their biological properties. Xanthone XH9, the most effective ROS and RNS scavenger, presented the lowest anodic potential voltage $\left(E_{p a}=0.15 \mathrm{~V}\right.$ at $\left.\mathrm{pH} 7.4 \mathrm{vs} \mathrm{Ag} / \mathrm{AgCl}\right) .{ }^{[9]}$

Mass spectrometry (MS) is one of the most sensitive and selective methods for molecular analysis and has the potential to yield structural information about individual molecules in a complex mixture in addition to their molecular mass. Also, product ion spectra (MS/MS) data, that can be effortlessly generated, yields important structural information via interpretation of the fragmentation pathways. The characterization of dietary polyphenols, namely xanthones, their metabolites and their in vivo description, in what concerns their clinical pharmacokinetic and pharmacodynamics profile, has been performed using MS techniques together with other analytical methods. ${ }^{[10]}$

The potential relationship between radical-mediated xanthone chemistry in the gas phase and their promising antioxidant activities has not been previously explored. Therefore, it was considered to investigate, for the first time, the mass spectral fragmentation behaviour of nine 2,3diarylxanthones bearing up to four hydroxyl groups at various sites on the two aryl rings (Fig. 1) using electrospray ionization tandem mass spectrometry (ESI-MS/MS). It was considered that xanthones containing (a) one hydroxyl group (XH2 and $\mathrm{XH} 4)$; (b) two hydroxyl groups (XH3, XH5 and $\mathrm{XH7}$ ); (c) three hydroxyl groups (XH6 and XH8); (d) four hydroxyl groups (XH9); and (e) the non-substituted XH1 could be differentiated by ESI-MS/MS and that detailed structural information could be obtained relevant to understand their antioxidant properties and help predict the potential biological activity of new xanthones.

\section{EXPERIMENTAL}

Xanthone XH1 and the hydroxy-2,3-diarylxanthones XH2-9 were synthesized via a five-step chemical sequence previously described in the literature. ${ }^{[5]}$ The purity of all synthesized compounds was assessed by 1D and 2D nuclear magnetic resonance (NMR) spectroscopy, high-resolution mass spectrometry (HRMS) and elemental analysis.

ESI-MS and collision-induced dissociation tandem mass (CID-MS/MS) spectra of the studied compounds were acquired using a Q-TOF 2 instrument (Micromass, Manchester, UK). Solutions of the samples with a concentration of $1 \mathrm{mg} / \mathrm{mL}$ were prepared dissolving the compound with a 1:1 mixture of $\mathrm{MeOH} / \mathrm{CHCl}_{3}$. Samples for ESI analysis were prepared by diluting $2 \mu \mathrm{L}$ of the xanthone solutions with $200 \mu \mathrm{L}$ of methanol/formic acid $(0.1 \%)$. Nitrogen was used as nebulizer gas and argon was used as collision gas. Samples were introduced into the mass spectrometer at a flow rate of $10 \mu \mathrm{L} / \mathrm{min}$, the needle voltage was set at $3000 \mathrm{~V}$, with the ion source at $80^{\circ} \mathrm{C}$ and desolvation temperature of $150^{\circ} \mathrm{C}$. The spectra were acquired for a cone voltage of $30 \mathrm{~V}$ to obtain good signal-to-noise ratio, either 
for the precursor or the product ions of interest. CID-MS/MS spectra were acquired by selecting the desired ion with the quadrupole section of the mass spectrometer and using collision energies of 30, 35 and $40 \mathrm{eV}$. The product ions were analysed with the time-of-flight (TOF) analyser. In MS and MS/MS experiments, TOF resolution was set to approximately 9000 (FWHM). Data acquisitions were carried out with a Micromass MassLynx 4 data system.

\section{Statistical analysis}

Principal Component Analysis (PCA) was applied to the raw data depicted in Fig. 2. ${ }^{[11]} \mathrm{A}$ biplot was built in order to facilitate the interpretation of the relationships between observations (compounds) and variables (most significant product ions). A multiple regression procedure was applied to the previous dataset as a function of the antioxidant activity expressed as $\mathrm{E}_{p a}$ and ORAC values.

\section{RESULTS AND DISCUSSION}

Xanthone XH1 and the hydroxy-2,3-diarylxanthones (XH2-9) studied by ESI-MS are shown in Fig. 1. Under the ionization conditions described above the $[\mathrm{M}+\mathrm{H}]^{+}$ions are formed (Table 1). The protonated molecules were dissociated by collision with a gas and the fragmentation pattern observed was used to identify typical ions that will be amenable to fingerprint each specific structural feature of the studied compounds.

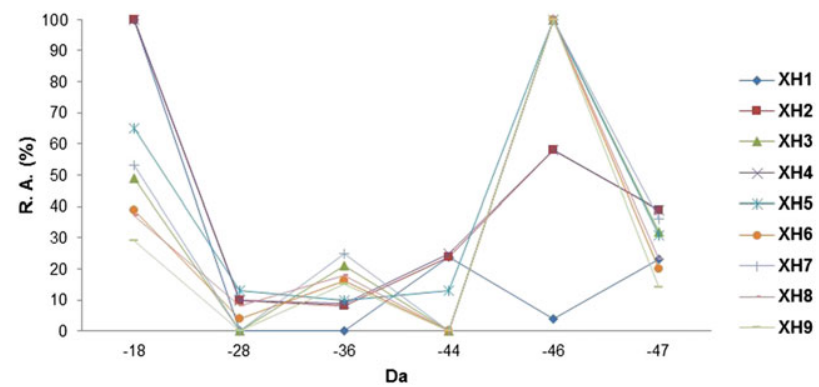

Figure 2. Product ions formed for compounds $\mathrm{XH1}-9$ by the characteristic losses of $18 \mathrm{Da}, 28 \mathrm{Da}, 36 \mathrm{Da}, 44 \mathrm{Da}, 46 \mathrm{Da}$ and $47 \mathrm{Da}$ versus their relative abundances obtained at a collision energy of $40 \mathrm{eV}$.
The ESI CID-MS/MS spectra of the $[\mathrm{M}+\mathrm{H}]^{+}$ions of XH1-9 were acquired using three different collision energies, namely 30, 35 and $40 \mathrm{eV}$. In the ESI CID-MS/MS spectra obtained at a collision energy of $30 \mathrm{eV}$, the intact cations $[\mathrm{M}+\mathrm{H}]^{+}$represent the base peak for $\mathrm{XH1}-9$ and fewer product ions are observed (data not shown). When the collision energy was set to 35 and $40 \mathrm{eV}$ the obtained spectra were altogether similar and the intact protonated molecules $[\mathrm{M}+\mathrm{H}]^{+}$were observed in a low relative abundance ranging from 7 to $35 \%$. In general, a higher degree of fragmentation was observed in these conditions; thus we used in these studies the MS/MS data obtained with a collision energy of $40 \mathrm{eV}$ which are summarized in Supplementary Table S1 (Supporting Information). The tandem mass spectra (ESI-MS/MS) of the $[\mathrm{M}+\mathrm{H}]^{+}$ions of compounds XH1, XH4, XH6 and XH9 are shown in Fig. 3 as examples.

Considering a collision energy of $40 \mathrm{eV}$, the major product ion (base peak of the MS/MS spectrum) for XH1, XH2 and $\mathrm{XH} 4$ corresponded to the $\left[(\mathrm{M}+\mathrm{H})-\mathrm{H}_{2} \mathrm{O}\right]^{+}$ions while for $\mathrm{XH} 3$ and $\mathrm{XH} 5-9$ the most abundant ion detected corresponded to the $\left[(\mathrm{M}+\mathrm{H})-\left(\mathrm{H}_{2} \mathrm{O}+\mathrm{CO}\right)\right]^{+}$ions.

Figure 2 represents the relative abundances of the most significant product ions (loss of $18 \mathrm{Da}, 28 \mathrm{Da}, 36 \mathrm{Da}, 44 \mathrm{Da}$, $46 \mathrm{Da}$ and $47 \mathrm{Da}$ from the precursor $[\mathrm{M}+\mathrm{H}]^{+}$ions) obtained for all the compounds. The collected data (Supplementary Table S1, Supporting Information) showed that there are only three fragmentation pathways that are common to all the xanthones studied, specifically, the loss of $\mathrm{H}_{2} \mathrm{O}(18 \mathrm{Da})$, concomitant loss of $\mathrm{H}_{2} \mathrm{O}$ plus ( $\left.\mathrm{HCO}\right)^{\bullet}(47 \mathrm{Da})$, and combined loss of $\mathrm{C}_{2} \mathrm{H}_{3} \mathrm{O}^{\bullet}$ plus $\mathrm{CO}(71 \mathrm{Da})$. Considering xanthones XH2-9, that possess different patterns of hydroxyl substituents in their structure, the common fragmentation pathways are the losses of $36 \mathrm{Da}, 46 \mathrm{Da}, 65 \mathrm{Da}, 74 \mathrm{Da}$ and $89 \mathrm{Da}$. Several other less significant fragmentations, in terms of relative abundance, are observed randomly among all the xanthone spectra. To understand how the structural features of each xanthone may rule the fragmentation pathways and their biological profile, this study was performed by analysing firstly the MS/MS data of the non-substituted xanthone XH1.

The spectrum obtained for XH1 (Fig. 3) showed the lowest number of product ions of the MS/MS spectra of this family of studied compounds XH1-9 (Supplementary Table S1, Supporting Information). The product ion formed by loss of $18 \mathrm{Da}\left(\mathrm{H}_{2} \mathrm{O}\right)$ is produced for $\mathrm{XH} 1$ in high relative abundance $(100 \%$ RA) and therefore constitutes a rather important fragmentation pathway for this compound. This same observation can be made for the isomeric group formed by $\mathrm{XH} 2$ and $\mathrm{XH} 4$, possessing one phenolic group. The

Table 1. Xanthones XH1-9 studied by mass spectrometry and calculated monoisotopic masses of the $[\mathrm{M}+\mathrm{H}]^{+}$ions

\begin{tabular}{|lllllc|}
\hline Compd. & $\mathrm{R}^{1}$ & $\mathrm{R}^{2}$ & $\mathrm{R}^{3}$ & $\mathrm{R}^{4}$ & Monoisotopic mass of $[\mathrm{M}+\mathrm{H}]^{+}(\mathrm{Da})$ \\
\hline XH1 & $\mathrm{H}$ & $\mathrm{H}$ & $\mathrm{H}$ & $\mathrm{H}$ & 349.1223 \\
XH2 & $\mathrm{OH}$ & $\mathrm{H}$ & $\mathrm{H}$ & $\mathrm{H}$ & 365.1172 \\
XH3 & $\mathrm{OH}$ & $\mathrm{OH}$ & $\mathrm{H}$ & $\mathrm{H}$ & 381.1121 \\
XH4 & $\mathrm{H}$ & $\mathrm{H}$ & $\mathrm{OH}$ & $\mathrm{H}$ & 381.1172 \\
XH5 & $\mathrm{OH}$ & $\mathrm{H}$ & $\mathrm{OH}$ & $\mathrm{H}$ & 397.1071 \\
XH6 & $\mathrm{OH}$ & $\mathrm{OH}$ & $\mathrm{OH}$ & $\mathrm{H}$ & 381.1121 \\
XH7 & $\mathrm{H}$ & $\mathrm{H}$ & $\mathrm{OH}$ & $\mathrm{OH}$ & 397.1071 \\
XH8 & $\mathrm{OH}$ & $\mathrm{H}$ & $\mathrm{OH}$ & $\mathrm{OH}$ & 413.1020 \\
XH9 & $\mathrm{OH}$ & $\mathrm{OH}$ & $\mathrm{OH}$ & $\mathrm{OH}$ & \\
\hline
\end{tabular}




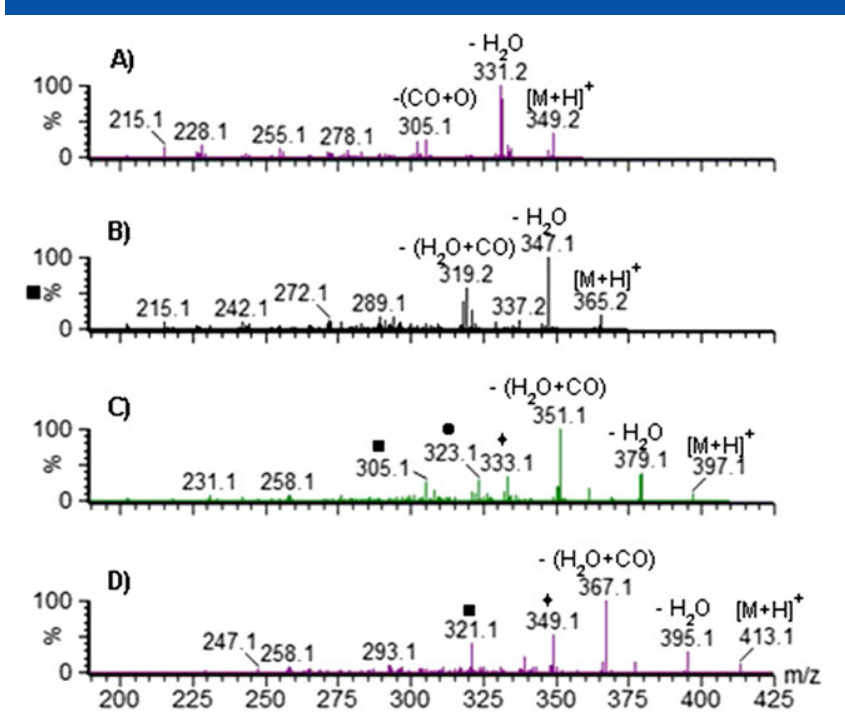

Figure 3. Product ion spectra (CID-MS/MS) of the selected precursor ions $[\mathrm{M}+\mathrm{H}]^{+}:(\mathrm{A})$ at $m / z 349$ for $\mathrm{XH} 1$; (B) at $m / z 365$ for XH4; (C) at $m / z 397$ for XH6; and (D) at $m / z 413$ for XH9. $\bullet,[\mathrm{M}+\mathrm{H}-64]^{+} ; \boldsymbol{\bullet},[\mathrm{M}+\mathrm{H}-74]^{+} ; \mathbf{0},[\mathrm{M}+\mathrm{H}-92]^{+}$.

mechanism for this loss is proposed in Scheme 1 and considers the site of protonation for these compounds in the oxygen of the heterocycle and formation of the stable carbocation $1 \mathrm{~b}, 2 \mathrm{~b}$ and $4 \mathrm{~b}(\mathrm{~m} / \mathrm{z} 331$ and 347$)$. This mechanism can also be considered for the remaining xanthones studied which also showed the formation of this product ion but in comparatively lower relative abundance.
The rather interesting loss of $47 \mathrm{Da}$, detected for all the studied xanthones independently of the pattern of substitution at ring $\mathrm{D}$ or $\mathrm{E}$, leads to the formation of product ions ranging in relative abundances from 14 to $44 \%$ (Scheme 1 and Supplementary Table S1, Supporting Information). These product ions formed by loss of $47 \mathrm{Da}\left[\mathrm{H}_{2} \mathrm{O}+(\mathrm{HCO})^{\bullet}\right]$ can also be justified through formation of carbocation $1 \mathrm{~b}, 2 \mathrm{~b}$ and $4 \mathrm{~b}$ that subsequently loses a fragment corresponding to $(\mathrm{HCO})^{\bullet}$. An intramolecular rearrangement that leads to a ring contraction would end in the formation of product ion $1 \mathrm{c}, 2 \mathrm{c}$ and $4 \mathrm{c}(\mathrm{m} / \mathrm{z} 302$ and 318$)$ as proposed in Scheme 1 . The spectra of the isomeric group $\mathrm{XH} 2$ and $\mathrm{XH} 4$ showed the highest relative abundances for these product ions $(2 \mathrm{c}$ and $4 \mathrm{c}$ at $\mathrm{m} / \mathrm{z} 318$, Scheme 1), 44\% and 39\% RA, respectively.

The product ion formed by loss of 47 Da can additionally lose another molecule of $\mathrm{H}_{2} \mathrm{O}$, as exemplified in Scheme 1 for compound $\mathrm{XH} 2$ with formation of product ion $2 \mathrm{~d}$ at $m / z 300$ (combined neutral loss of $\left.\left[2 \mathrm{H}_{2} \mathrm{O}+(\mathrm{HCO})^{\bullet}\right]\right)$. This second loss of water is observed for all the xanthones studied with relative abundances ranging from 7 to $16 \%$, except for $\mathrm{XH} 1$. This exception is related to the lack of the necessary hydroxyl substituent in either ring $\mathrm{E}$ or $\mathrm{D}$ as shown in Scheme 1 for $\mathrm{XH} 2$.

The CID-MS/MS spectrum obtained for XH1 also revealed some unique product ions characteristic of this nonsubstituted xanthone (Fig. 3(A)). These are the product ions formed through loss of $2 \mathrm{Da}, 15 \mathrm{Da}$, and $16 \mathrm{Da}$ obtained with relatively low abundances ranging from 10 to $17 \%$ (Supplementary Table S1, Supporting Information). The formation of product ion $1 \mathrm{~d}$ at $\mathrm{m} / \mathrm{z} 347$ (fragmentation pathway depicted in Scheme 1), only observed for XH1, occurs
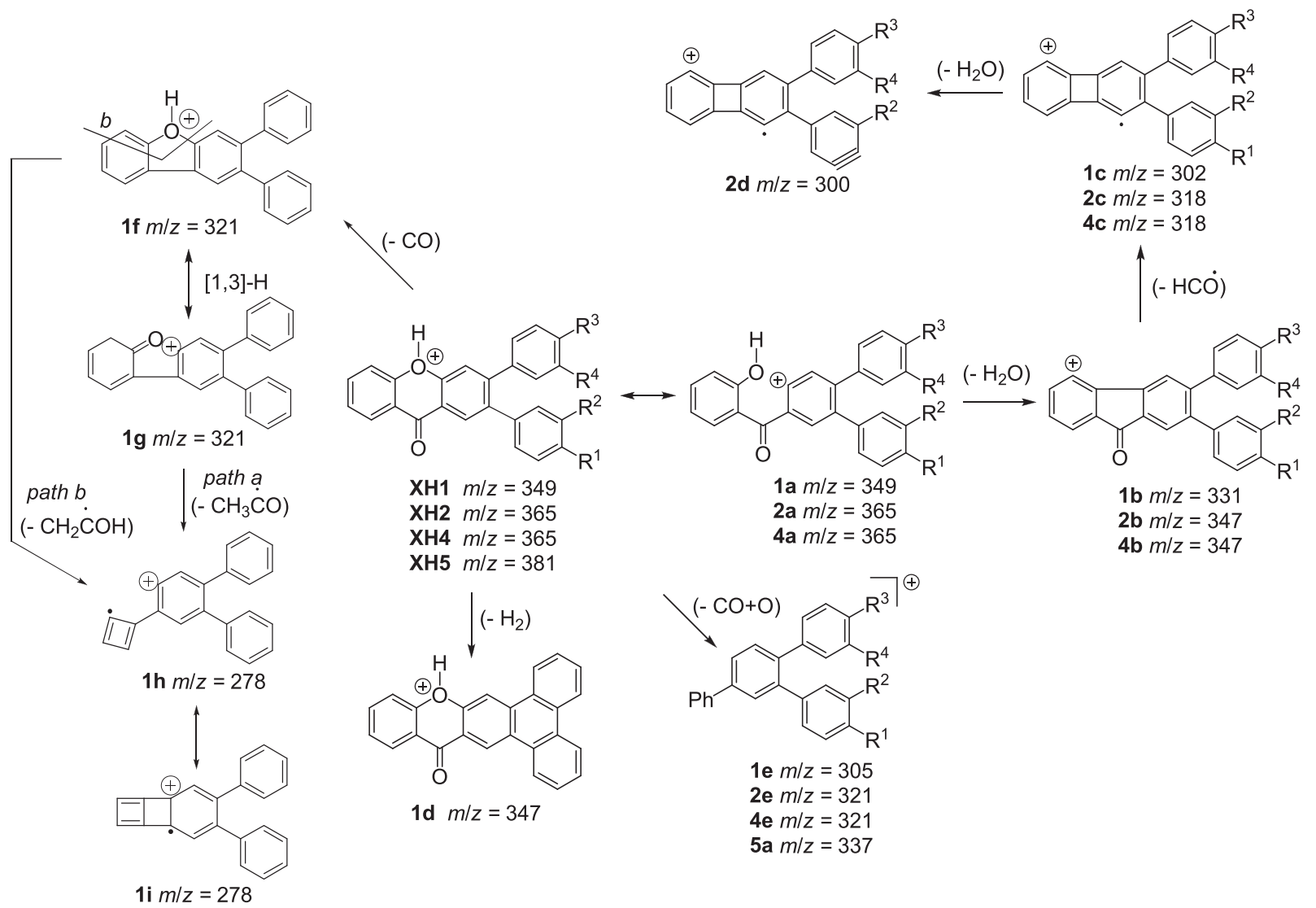

Scheme 1. Proposed mechanisms for the loss of $2 \mathrm{Da}, 18 \mathrm{Da}, 44 \mathrm{Da}, 47 \mathrm{Da}, 65 \mathrm{Da}$ and $71 \mathrm{Da}$. 
most likely due to the formation of a rather stable fused tetracyclic heterocyclic through an electrocyclisation reaction followed by elimination of a molecule of hydrogen (2 Da). This loss, although unusual (particularly considering the precursor molecules to be aromatic compounds, expected to be less prone to lose $\mathrm{H}_{2}$ ), has been observed in previous studies considering the characterization of $(E)$-2-styrylchromones by ESI-MS leading to the formation of benzo[a]xanthones. ${ }^{[12]}$

The neutral loss of $\mathrm{CO}$ plus $\mathrm{O}$ (44 Da) is only detected in the ESI CID-MS/MS spectra of the non-substituted XH1, and in compounds possessing hydroxyl groups at para positions of the 2- and/or 3-phenyl rings of the xanthone core: $\mathrm{XH} 2\left(\mathrm{R}^{1}=\mathrm{OH}\right)$, $\mathrm{XH} 4\left(\mathrm{R}^{3}=\mathrm{OH}\right)$ and $\mathrm{XH} 5\left(\mathrm{R}^{1}=\mathrm{R}^{3}=\mathrm{OH}\right)$. This loss leads to the formation of product ions with relative abundance ranging from 13 to $27 \%$. This product ion is thought to be formed through an intramolecular rearrangement that leads to a ring contraction to form the product ions 1e, 2e, $4 \mathrm{e}$ and $5 \mathrm{a}$ as proposed in Scheme 1. This loss has been previously observed in the negative CID of xanthones isolated from Swertia punicea using high-performance liquid chromatography and also in a comparative study of the common flavonoid subtypes, including xanthones, under unified conditions. ${ }^{[13]}$

Also common to all the studied xanthones is the product ion formed by loss of $71 \mathrm{Da}$. This product ion is obtained with relative abundances ranging from 7 to 18\% (Supplementary Table S1, Supporting Information) and results from the combined loss of $\left(\mathrm{C}_{2} \mathrm{H}_{3} \mathrm{O}\right)^{\bullet}$ plus $\mathrm{CO}$. This fragmentation pathway can be rationalized through formation of $1 \mathrm{f}$ by loss of $\mathrm{CO}$ from the endocyclic keto group with subsequent ring opening of the xanthone core and [1,3]-hydrogen shift leading to the formation of $1 \mathrm{~g}$ as depicted in Scheme 1 for $\mathrm{XH} 1$.
Elimination of an acetyl radical (pathway a) from structure $1 \mathrm{~g}$ leads to the observed product ion 1i. A different pathway can be disclosed considering the loss of a vinyl radical (pathway b) leading also to the formation of $1 \mathrm{i}$ (Scheme 1).

A different fragmentation pathway can be proposed for the loss of $\mathrm{H}_{2} \mathrm{O}$ involving a hydroxyl group at ring $\mathrm{D}$ or $\mathrm{E}$, considering once more the protonation at the oxygen of the heterocyclic ring (Scheme 2). The mechanism shown in Scheme 2 is exemplified for XH6 with formation of product ion $6 a$ at $m / z 379$ in a relative abundance of $39 \%$. This fragmentation mechanism cannot be envisioned for $\mathrm{XH} 1$ for the obvious reason that it does not have a hydroxyl group in its structure. This product ion can further fragmented by loss of the carbonyl group leading to a concomitant loss of $46 \mathrm{Da}$ $\left(\mathrm{H}_{2} \mathrm{O}\right.$ plus $\left.\mathrm{CO}\right)$ and formation of the most abundant product ion $6 \mathrm{~b}$ at $\mathrm{m} / \mathrm{z} 351$ in the CID-MS/MS spectrum of XH6. This concomitant loss of $\mathrm{H}_{2} \mathrm{O}$ plus $\mathrm{CO}$ represents a well-known fragmentation for a range of flavonoids. ${ }^{[14]}$ Interestingly, the loss of $46 \mathrm{Da}$ is found in the spectra of all the studied xanthones except for the non-substituted XH1 which supports the necessary involvement of a hydroxyl group at ring $\mathrm{D}$ and/or $\mathrm{E}$ in this fragmentation pathway. The loss of $46 \mathrm{Da}$ can be justified by a different mechanism that suggests the site of protonation in the carbonyl group of the heterocyclic ring. A fragmentation pathway for this loss has been proposed in Scheme 3 using as example $\mathrm{XH} 2$. Through the formation of $2 \mathrm{i}$ (pathway a) or $2 \mathrm{k}$ (pathway b) one could also consider the subsequent loss of $28 \mathrm{Da}(\mathrm{CO})$ with formation of product ion $2 \mathrm{j}$ or 21 at $\mathrm{m} / \mathrm{z} 319$ (Scheme 3). This fragmentation pathway does not justify however the observed loss of 47 Da. A mechanism proposing the site of protonation in the carbonyl

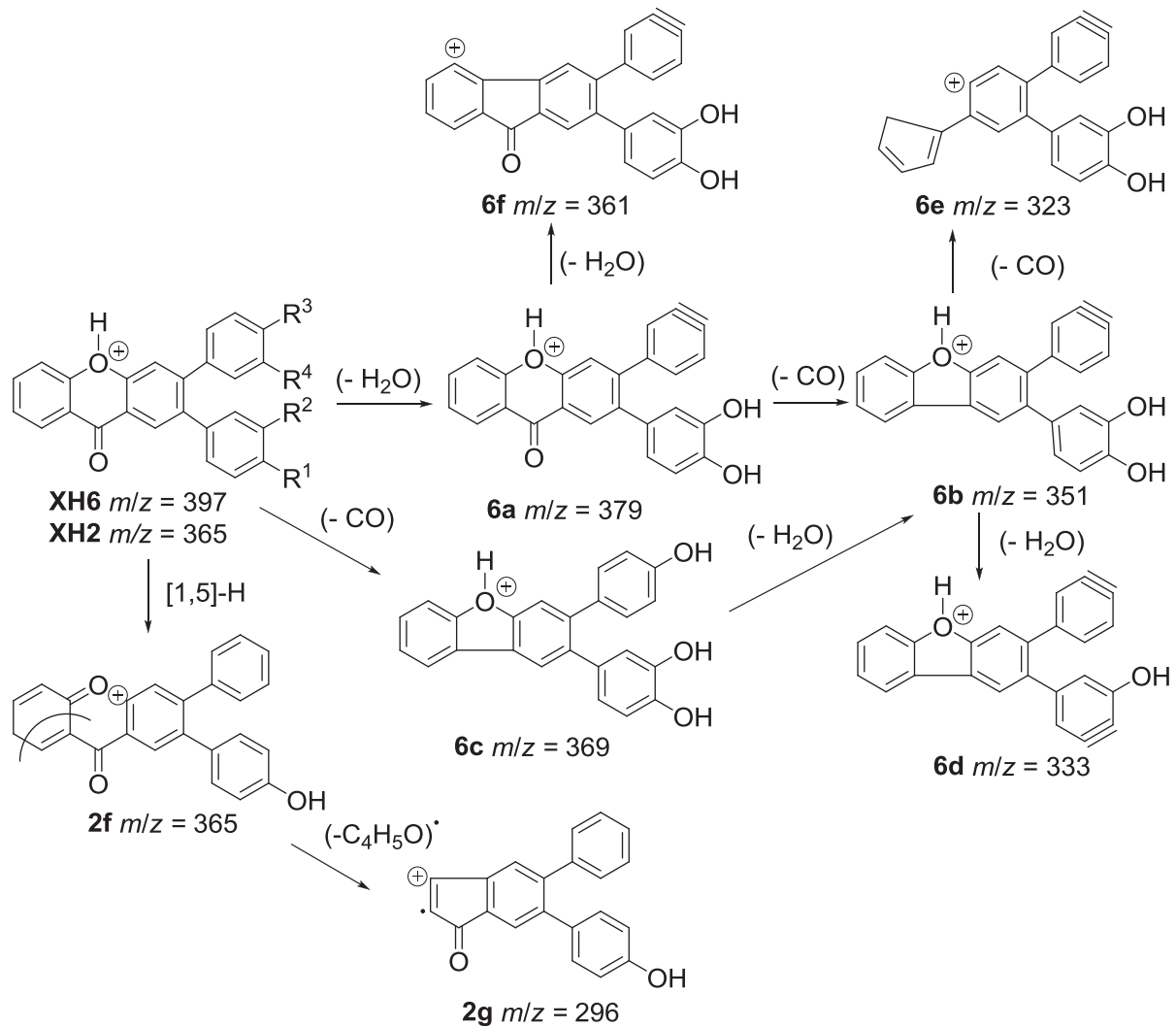

Scheme 2. Fragmentation pathways proposed for the combined losses of $\mathrm{H}_{2} \mathrm{O}$ and $\mathrm{CO}$ considering the protonation in the oxygen of the heterocycle. 


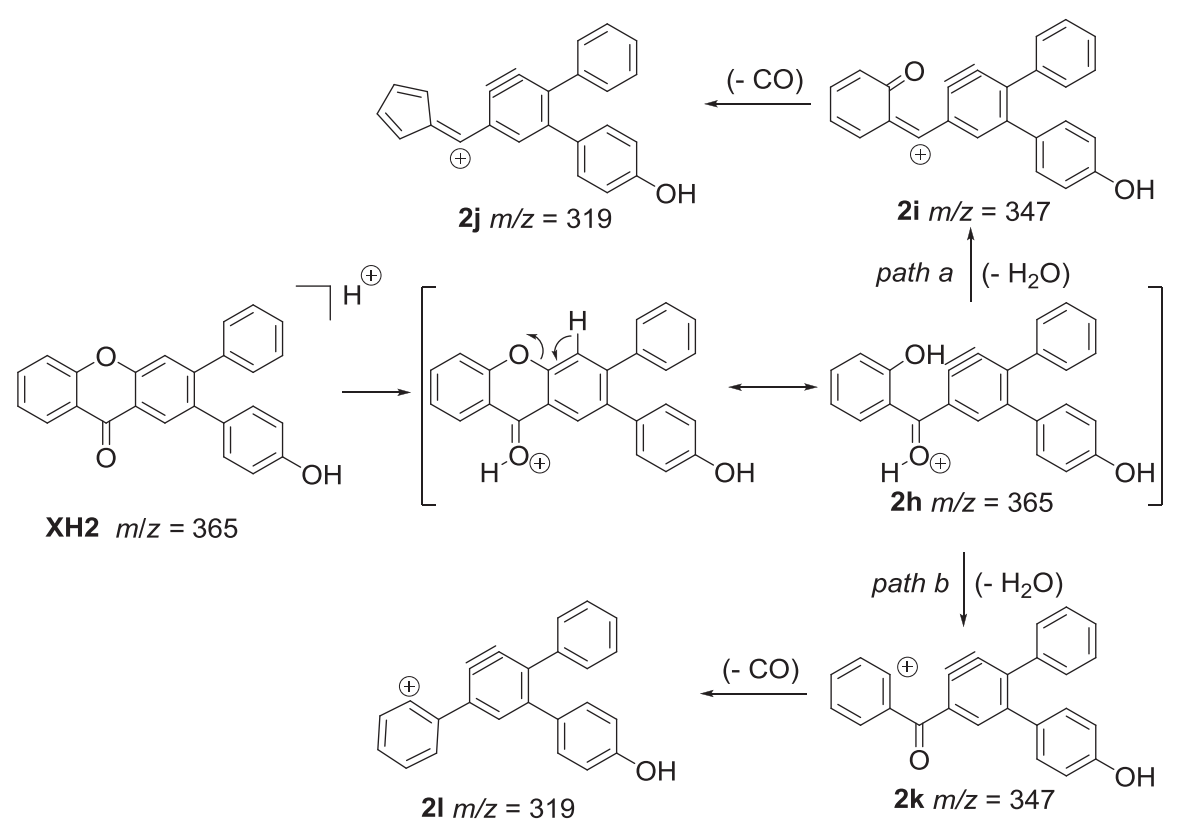

Scheme 3. Fragmentation pathways proposed for the loss of 46 Da considering the protonation in the carbonyl group of the heterocycle.

group of the heterocyclic ring has also been previously stated for the loss of 18 Da observed in the CID-MS/MS spectrum of $12 H$-benzo[12a]xanthen-12-one. ${ }^{[12]}$

As stated before, when considering xanthones $\mathrm{XH} 2-9$ other common fragmentation pathways comprise the losses of $36 \mathrm{Da}, 74 \mathrm{Da}$, and $89 \mathrm{Da}$. For instance, in the case of $\mathrm{XH}$, the loss of $36 \mathrm{Da}$ is justified in Scheme 2 through a subsequent second loss of $18 \mathrm{Da}\left(\mathrm{H}_{2} \mathrm{O}\right)$ from carbocation $6 \mathrm{a}$ leading to the formation of carbocation $6 \mathrm{f}$ at $\mathrm{m} / \mathrm{z} 361$. This loss leads to the formation of product ions with relative abundance ranging from 9 to 25\% (Supplementary Table S1, Supporting Information). The loss of $74 \mathrm{Da}$ leads to the formation of the product ion 6e (Scheme 2) through concomitant loss of two molecules of $\mathrm{CO}$ plus $\mathrm{H}_{2} \mathrm{O}$. The product ions formed by loss of $89 \mathrm{Da}$ have relative abundances that range from 7 to $15 \%$ (Supplementary Table S1, Supporting Information) and it was considered to be formed through the combined loss of $\mathrm{CO}$ plus $\left(\mathrm{C}_{2} \mathrm{H}_{3} \mathrm{O}\right)^{\bullet}$ (Scheme 1), with subsequent loss of one molecule of $\mathrm{H}_{2} \mathrm{O}$ from either a hydroxyl group at ring $\mathrm{D}$ and/or $\mathrm{E}$ which therefore is not observed for $\mathrm{XH} 1$.

Scheme 2 also depicts the formation of $6 \mathrm{~d}$ resulting from the combined loss of two molecules of $\mathrm{H}_{2} \mathrm{O}$ plus $\mathrm{CO}$ in a total of $64 \mathrm{Da}$. This fragmentation pathway is observed for the isomeric group $\mathrm{XH} 3$ and $\mathrm{XH} 7$ in low relative abundances (7 and 9\%, respectively), and for $\mathrm{XH} 6, \mathrm{XH} 8$ and $\mathrm{XH} 9$ in higher relative abundances (23, 43 and 51\%, respectively). The collected data revealed that this neutral loss is more favourable for highly substituted xanthones, non-existent in the CID-MS/MS spectra of XH1, XH2, XH4 and XH5 and results in product ions with low relative abundance for $\mathrm{XH} 3$ and $\mathrm{XH} 7$ which indicates that the second molecule of water results from an elimination of hydroxyl from the $\mathrm{D}$ or/and E rings. This neutral loss of $64 \mathrm{Da}$ can also be justified by an alternative fragmentation pathway that considers the combined loss of $\mathrm{H}_{2} \mathrm{O}$ and $(\mathrm{HCO})^{\bullet}$ plus ${ }^{\circ} \mathrm{OH}$.
The loss of $28 \mathrm{Da}(\mathrm{CO})$ is a common fragmentation pathway in the CID-MS/MS spectra of XH2, XH4, XH5 and XH8 that results in a product ion with low relative abundance (4-13\%). These ions are probably formed through cleavage of the protonated molecules leading to the formation of a five-membered ring (Scheme 2). The extrusion of $\mathrm{CO}$ from the chromone nucleus has also been described in the fragmentation of several chromone derivatives. ${ }^{[12,15]}$ This same fragmentation pathway is commonly observed in the CID-MS/MS spectra of flavonoids and was described as characteristic of their mass spectra when operating in the negative ion mode. ${ }^{[16]}$ This fragmentation pathway is not observed for the non-substituted $\mathrm{XH1}$, xanthones containing a catechol moiety in ring $\mathrm{D}$ or $\mathrm{E}, \mathrm{XH} 3$ and $\mathrm{XH} 7$, respectively, and xanthones XH6 and XH9.

The isomeric group formed by $\mathrm{XH} 2$ and $\mathrm{XH} 4$, which possess one phenol group, has under the same conditions indistinguishable spectra and could not be differentiated using the information collected by CID-MS/MS. Interestingly the product ions formed by loss of $69 \mathrm{Da}$, although formed in low relative abundance (Supplementary Table S1, Supporting Information), are unique to these two xanthones. The fragmentation pathway, leading to the formation of the product ion at m/z 296 in the MS/MS spectra of XH2 and $\mathrm{XH} 4$, is depicted in Scheme 2 for $\mathrm{XH} 2$ and comprises the ring opening of the xanthone core and a $[1,5]$-hydrogen shift leading to the formation of 21 . The loss of a radical fragment $\mathrm{C}_{4} \mathrm{H}_{5} \mathrm{O}^{\bullet}$ allows the formation of product ions $2 \mathrm{~m}$.

Other isomeric xanthones may be differentiated by comparison of their MS/MS spectra. In the case of xanthones having two hydroxyl groups, the ones possessing a catechol group, $\mathrm{XH} 3$ and $\mathrm{XH}$, can be differentiated from $\mathrm{XH} 5$ that has two phenol groups at positions 2 and 3 of the xanthone core. Comparing these three MS/MS spectra one could observe that the product ions formed through loss of $28 \mathrm{Da}$, $44 \mathrm{Da}, 93 \mathrm{Da}, 94 \mathrm{Da}, 139 \mathrm{Da}$, and $150 \mathrm{Da}$ are only observed 
for compound XH5. Also, the product ions formed through loss of $48 \mathrm{Da}, 63 \mathrm{Da}, 64 \mathrm{Da}$, and $123 \mathrm{Da}$ found in the spectra of compound $\mathrm{XH} 3$ and $\mathrm{XH} 7$ are absent in the spectrum of xanthone XH5. Another interesting feature found in these spectra is the fact that the product ion formed by loss of $121 \mathrm{Da}$ is observed for almost all the xanthones studied except for XH3 and XH7. The isomeric group formed by XH6 (Fig. 3(C)) and XH8, that have three hydroxyl groups, also presented identical MS/MS spectra; the only difference is in the relative abundance of the product ion formed by the loss of $64 \mathrm{Da}$. This ion at $m / z 333$ is formed for XH6 (Scheme 2) and XH8 in 23 and $43 \%$ RA, respectively. The corresponding product ion of $\mathrm{XH} 9$ is formed in 51\% RA and with lower relative abundance in XH3 (7\% RA) and XH7 (9\% RA).

Supplementary Table S1 (Supporting Information) summarizes the results obtained in the several biological assays performed for XH1-9 and the best results achieved are assigned in grey. The results gathered in S1 seem to relate the lack or general diminutive scavenging activity of $\mathrm{XH} 1, \mathrm{XH} 2$ and XH4 with the detection of the base peak ion $[\mathrm{M}+\mathrm{H}-18]^{+}$. All the other studied compounds, that showed a more promising biological profile, showed as base peak the product ion formed through combined loss of $\mathrm{H}_{2} \mathrm{O}$ plus $\mathrm{CO}(46 \mathrm{Da})$, as can be seen in Fig. 2. Also interesting is the neutral loss of $64 \mathrm{Da}$ which is not observed for $\mathrm{XH} 1, \mathrm{XH} 2, \mathrm{XH} 4$, and $\mathrm{XH}$. The product ion formed by loss of $64 \mathrm{Da}$ is observed for xanthones containing a catechol unit (XH3 and $\mathrm{XH} 6-9)$ and has the highest relative abundance (51\% RA) in the case of the most potent ROS and RNS scavenger XH9 that bears two of these moieties.

A PCA revealed that the first two principal components account for $98 \%$ of the total variability. It is interesting that the most relevant pattern that emerges from Fig. 4 is the distribution of $\mathrm{XH} 5$ to $\mathrm{XH} 9$ samples, crossing $\mathrm{XH} 3$ and $\mathrm{XH6}-8$, which is almost linear. Also worthy of mention is the fact that compound $\mathrm{XH} 5$ is in an opposite location to $\mathrm{XH} 9$ on the distribution represented in Fig. 4. Compound XH5, containing two phenol groups, is the most powerful scavengers of $\mathrm{ROO}^{\circ}$ providing a ORAC value of $2.88 \pm 0.09 \mathrm{M}$ while, in contrast,

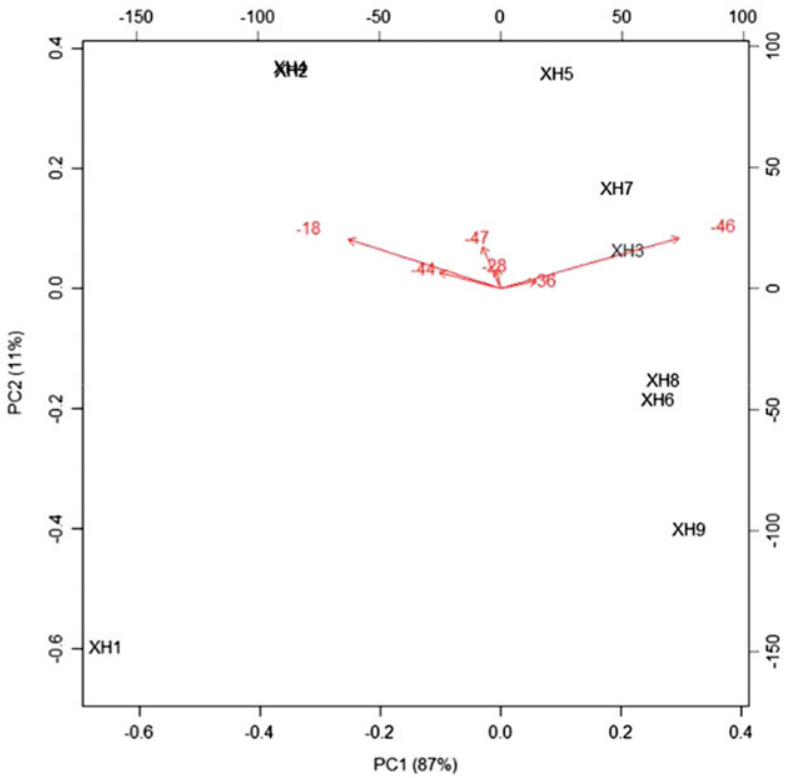

Figure 4. PCA biplot of relative abundances of the most relevant product ions.
$\mathrm{XH}$, that contains two catechol moieties, was the less active one presenting an ORAC value of $0.28 \pm 0.03 \mathrm{M}$ (Supplementary Table S1, Supporting Information). XH9 is, however, the compound studied with the highest scavenger activity. The importance of the catechol ring in these molecules has been documented and related previously with the highest scavenger activity observed for most of the tested ROS and RNS. ${ }^{[6]}$

The $\mathrm{ROO}^{\circ}$ scavenging activity of these compounds revealed to be more dependent on the hydroxylation pattern in the aryl group. ${ }^{[6]}$ In particular, the ORAC assay showed that the presence of a phenol group seems to be extremely important for the scavenging effect of these species and the best results were obtained for compounds containing this moiety: $\mathrm{XH}$, $\mathrm{XH} 4$ and $\mathrm{XH}$. Interestingly these compounds also show a unique combined loss of $\mathrm{CO}$ plus $\mathrm{O}$ in their CID-MS/MS spectra. The formation of this product ion is also observed for compound $\mathrm{XH} 1$ but this compound did not show any activity up to $1 \mu \mathrm{M}$, the highest tested concentration. Compound $\mathrm{XH} 1$ shows a totally different fragmentation pattern and the lowest biological activity observed when comparing with the other xanthones, which is clearly noted by the distribution shown in Fig. 4. The referred linear distribution shown in Fig. 4 ( $\mathrm{XH} 5$ to $\mathrm{XH} 9$ ) is mostly characterized by the loss of $47 \mathrm{Da}$ and, to a lesser extent, the loss of $28 \mathrm{Da}$ and $18 \mathrm{Da}$ which therefore seems to account for this referred linearity. The loss of $47 \mathrm{Da}$ is also common to $\mathrm{XH} 2$ and $\mathrm{XH} 4$, although having a higher RA (44-39\%) for the product ions of these compounds than the one observed for XH9 (14\%). The latter compounds, XH2 and XH4, are isomeric xanthones containing a phenol moiety in ring $\mathrm{D}$ or E, showing therefore the same behaviour.

Multiple regression models were built in order to assess the antioxidant activity using two surrogate variables (ORAC and $\mathrm{E}_{p a}$ ). The regression errors (expressed as the relative root mean square error) were found to be $17.2 \%$ and $1.2 \%$ for ORAC and $\mathrm{E}_{p a}$, respectively. Figure 5 depicts the relationship between the most relevant product ions and the $\mathrm{E}_{p a}(\mathrm{~V})$ values. The results suggest that there seems to be a strong relationship (adjusted $\mathrm{R}^{2}$ of 0.998 ) between the relevant product ions and the antioxidant activity (expressed by the $\mathrm{E}_{p a}$ values). However, the results are skewed towards the minimum and maximum values of $\mathrm{E}_{p a}$, which precludes a much robust evidence of the relationship.

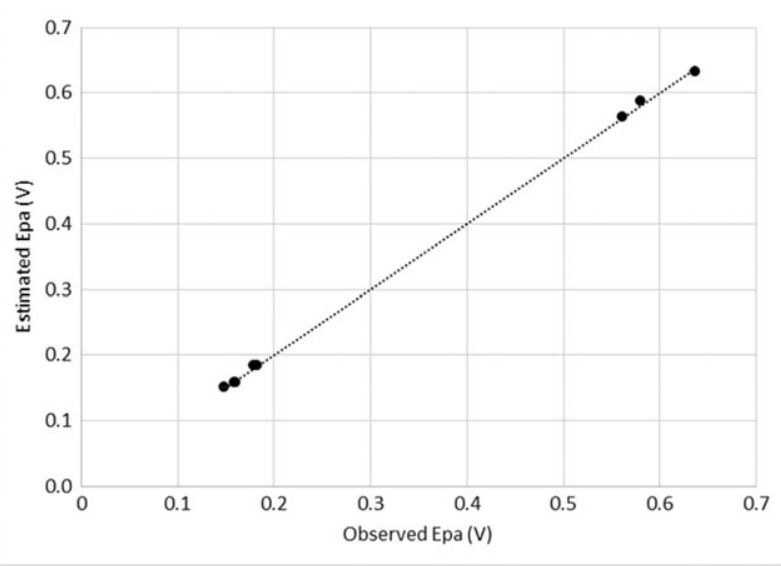

Figure 5. Relationship plot between observed $E_{p a}(V)$ and estimated $E_{p a}$ values using Chart 1 dataset. Adjusted $R^{2}$ (0.998) and a $p$-value of 0.034 . 


\section{CONCLUSIONS}

The work described herein reports the first systematic study involving the characterization by ESI-MS/MS of 2,3diarylxanthones by analysing their collision-induced dissociation and the fragmentation mechanisms of the protonated counterparts. From these studies, a clear relationship between some structural important features shown by these molecules, such as the catechol moiety, and their mass spectrometry profile was found.

The analysed compounds revealed under the same experimental conditions the formation of the product ion $[(\mathrm{M}+\mathrm{H})-18]^{+}$as base peak for $\mathrm{XH} 1, \mathrm{XH} 2$ and $\mathrm{XH} 4$ and product ion $[(\mathrm{M}+\mathrm{H})-46]^{+}$for $\mathrm{XH} 3$ and $\mathrm{XH} 5-9$. The concomitant loss of $\mathrm{H}_{2} \mathrm{O}$ plus $\mathrm{CO}$ corresponded therefore to the most important fragmentation pathway for the most promising xanthones, $\mathrm{XH} 3$ and $\mathrm{XH5}-9$, in what concerns their biological profile. Also, unique to this set of xanthones containing a catechol unit (XH3, XH5-9) is the product ion formed by loss of two molecules of $\mathrm{H}_{2} \mathrm{O}$ plus $\mathrm{CO}(64 \mathrm{Da})$. This product ion showed the highest relative abundance for the most potent ROS and RNS scavenger XH9 that contains two of these moieties. We have also showed that there is a strong relationship between some of the biological activities of the studied 2,3diarylxanthones and of their ESI-MS/MS fragmentation spectra. These findings were also confirmed by statistical multivariate analysis. The multivariate statistical analysis of the results, computed by the application of principal component analysis and multiple regression, gives a strong suggestion that the recovered mass spectrometry features are important surrogate's traits of biological activities for this family of compounds, allowing a cheaper and more convenient initial screening of these types of molecules.

\section{Acknowledgements}

Thanks are due to Fundação para a Ciência e Tecnologia (FCT, Portugal), European Union, QREN, FEDER and COMPETE, for funding the QOPNA research unit (project PEst-C/QUI/ UI0062/2013; FCOMP-01-0124-FEDER-037296), and the Portuguese National Mass Spectrometry Network, RNEM (REDE/1504/REM/2005). E. M. P. Silva is also grateful to FCT (ref SFRH/BPD/66961/2009) for a Post-Doc grant.

\section{REFERENCES}

[1] K. Hostettman, M. Hostettman. Methods in plant biochemistry, in Plant Phenolics, vol. 1, (Eds: P. M. Dey, J. B. Harbone). Academic Press, 1989, p. 493.

[2] (a) J. C. Roberts. Naturally occurring xanthones. Chem. Rev. 1961, 38, 591; (b) L. Gales, A. M. Damas. Xanthones - A structural perspective. Curr. Med. Chem. 2005, 12, 2499.

[3] M. M. Pinto, M. E. Sousa, M. S. Nascimento. Xanthone derivatives: New insights in biological activities. Curr. Med. Chem. 2005, 12, 2517.

[4] S. S. Panda, M. Chand, R. Sakhuja, S. C. Jain. Xanthones as potential antioxidants. Curr. Med. Chem. 2013, 20, 4481.

[5] C. M. M. Santos, A. M. S. Silva, J. A. S. Cavaleiro. Efficient syntheses of new polyhydroxylated 2,3-diaryl-9H-xanthen9-ones. Eur. J. Org. Chem. 2009, 16, 2642.
[6] C. M. M. Santos, M. Freitas, D. Ribeiro, A. Gomes, A. M. S. Silva, J. A. S. Cavaleiro, E. Fernandes. 2,3Diarylxanthones as strong scavengers of reactive oxygen and nitrogen species: a structure/activity study. Bioorg. Med. Chem. 2010, 18, 6776.

[7] C. M. M. Santos, A. M. S. Silva, P. Filipe, R. Santus, L. K. Patterson, J.-C. Mazière, J. A. S. Cavaleiro, P. Morlière. Structure-activity relationships in hydroxy-2,3diarylxanthone antioxidants. Fast kinetics spectroscopy as a tool to evaluate the potential for antioxidant activity in biological systems. Org. Biomol. Chem. 2011, 9, 3965.

[8] P. Morlière, L. K. Patterson, C. M. M. Santos, A. M. S. Silva, J.-C. Mazière, P. Filipe, A. Gomes, E. Fernandes, M. B. Q. Garcia, R. Santus. The dependence of $\alpha$-tocopheroxyl radical reduction by hydroxy-2,3diarylxanthones on structure and micro-environment. Org. Biomol. Chem. 2012, 10, 2068.

[9] C. M. M. Santos, M. B. Q. Garcia, A. M. S. Silva, R. Santus, P. Morlière, E. Fernandes. Electrochemical characterization of bioactive hydroxyxanthones by cyclic voltammetry. Tetrahedron Lett. 2013, 54, 85.

[10] Selected examples: (a) R. Feng, Y.-Y. Zhang, X. Chen, Y. Wang, J.-G. Shi, C.-T. Che, J. H. K. Yeung, J.-Y. Ma, X.-S. Tan, C. Yang, Y.-L. Deng, Y.-K. Zhang. In vitro study on metabolite profiles of bioactive xanthones isolated from Halenia elliptica D. Don by high performance liquid chromatography coupled to ion trap time-of-flight mass spectrometry. J. Pharmaceut. Biomed. 2012, 62, 228; (b) J. D. van der Merwe, E. Joubert, M. Manley, D. de Beer, C. J. Malherbe, W. C. A. Gelderblom. Mangiferin glucuronidation: important hepatic modulation of antioxidant activity. Food Chem. Toxicol. 2012, 50, 808; (c) H. Liu, K. Wang, Y. Tang, Z. Sun, L. Jian, Z. Li, B. Wu, C. Huang. Structure elucidation of in vivo and in vitro metabolites of mangiferin. J. Pharmaceut. Biomed. 2011, 55, 1075; (d) F. Cai, W. Xu, H. Wei, L. Sun, S. Gao, Q. Yang, J. Feng, F. Zhang, W. Chen. Simultaneous determination of active xanthone glycosides, timosaponins and alkaloids in rat plasma after oral administration of Zi-Shen pill extract for the pharmacokinetic study by liquid chromatography-tandem mass spectrometry. J. Chromatogr. B 2010, 878, 1845.

[11] I. T. Jolliffe. Principal Component Analysis, (2nd edn). Springer, 2002.

[12] E. M. P. Silva, M. R. M. Domingues, A. M. S. Silva, J. A. S. Cavaleiro. Characterisation of $(E)$-2-styrylchromones by electrospray ionisation mass spectrometry: singular gasphase formation of benzoxanthenones. Rapid Commun. Mass Spectrom. 2012, 26, 2251.

[13] a) X.-G. Du, W. Wang, Q.-Y. Zhang, J. Cheng, B. Avula, I. A. Khan, D.-A. Guo. Identification of xanthones from Swertia punicea using high-performance liquid chromatography coupled with electrospray ionization tandem mass spectrometry. Rapid Commun. Mass Spectrom. 2012, 26, 2913; (b) W.-Z. Yang, M. Ye, X. Qiao, Q. Wang, T. Bo, D.-A. Guo. Collision-induced dissociation of 40 flavonoid aglycones and differentiation of the common flavonoid subtypes using electrospray ionization ion-trap tandem mass spectrometry and quadrupole time-of-flight mass spectrometry. Eur. J. Mass Spectrom. 2012, 18, 493.

[14] Selected examples: (a) Y. L. Ma, Q. M. Li, H. van den Heuvel, M. Claeys. Characterization of flavone and flavonol aglycones by collision-induced dissociation tandem mass spectrometry. Rapid Commun. Mass Spectrom. 1997, 11, 1357; (b) C. W. Huck, M. R. Buchmeiser, G. K. Bonn. Fast analysis of flavonoids in plant extracts by liquid chromatographyultraviolet absorbance detection on poly(carboxylic acid)coated silica and electrospray ionization tandem mass 
spectrometric detection. J. Chromatogr. A 2001, 943, 33; (c) F. Kuhn, M. Oehme, F. Romero, E. Abou-Mansour, R. Tabacchi. Differentiation of isomeric flavone/isoflavone aglycones by $\mathrm{MS}^{2}$ ion trap mass spectrometry and a double neutral loss of CO. Rapid Commun. Mass Spectrom. 2003, 17, 1941; (d) D. Tsimogiannis, M. Samiotaki, G. Panayotou, V. Oreopoulou. Characterization of flavonoid subgroups and hydroxy substitution by HPLC-MS/MS. Molecules 2007, 12, 593.

[15] T. Fossen, Ø. M. Andersen. Spectroscopic techniques applied to flavonoids, in Flavonoids. Chemistry, Biochemistry and Applications, (Eds: Ø. M. Andersen, K. R. Markham). CRC Press, Taylor \& Francis, Boca Raton, 2006.
[16] (a) N. Fabre, I. Rustan, E. Hoffmann, J. Quetin-Leclercq. Determination of flavone, flavonol, and flavanone aglycones by negative ion liquid chromatography electrospray ion trap mass spectrometry. J. Am. Soc. Mass Spectrom. 2001, 12, 707; (b) W. Wu, Z. Liu, F. Song, S. Liu. Structural analysis of selected characteristic flavones by electrospray tandem mass spectrometry. Anal. Sci. 2004, 20, 1103.

\section{SUPPORTING INFORMATION}

Additional supporting information may be found in the online version of this article at the publisher's website. 